\title{
Fetal Neurobehavioral Development: The Role of Maternal Psychosocial, Pathological, and Pharmacological Stress
}

\author{
Trenten Fenster ${ }^{1}$ (D) ${ }^{a}$, Mauna Rao ${ }^{1}$ (D) ${ }^{b}$, Yakov Mamzhi ${ }^{1}$, Harry Tsou Jr ${ }^{1}$ (D) ${ }^{d}$ \\ ${ }^{1}$ School of Medicine, Georgetown University \\ Keywords: development, neurobehavioral, fetal, maternal stressors \\ https://doi.org/10.52504/001c.12642
}

\section{Georgetown Medical Review}

Vol. 4, Issue 1, 2020

\begin{abstract}
Maternal-fetal stressors during the gestational period, such as psychosocial stress, disease burden, and medication use, have been shown to significantly affect the neurological and biological development of the fetus. To our knowledge, no previous study in the literature has synthesized the role of these various stressors in neurodevelopment into a single concise review article. Maternal psychosocial stress has been shown to raise levels of stress hormones, such as corticotropinreleasing hormone and adrenocorticotropic hormone, which in turn signal the release of glucocorticoids (eg, cortisol) along with catecholamines in the mother and fetus. These cascades could potentially have significant effects on fetal neurodevelopment. Further, this article highlights that certain maternal infectious disease states, such as influenza and Toxoplasma gondii, are associated with increased risk of psychiatric disorders among offspring, including schizophrenia and neurocognitive delay. Investigators have also found that antibodies from autoimmune disease have direct neurotoxic effects on neural cell receptors, manifesting in future cognitive performance deficits. Additionally, we note that the effects of opioid analgesics on fetal neurodevelopment are not well elucidated but some existing literature has found increased rates of neural tube defects and delays in central nervous system development. In summary, there is a need for increased prenatal screening for a wide breadth of maternal stressors to mitigate negative effects on fetal neurodevelopment.
\end{abstract}

\section{Introduction}

According to the fetal origins hypothesis, the fetal environment in utero can influence the health of the fetus, the future baby, and even the adult. ${ }^{1}$ In this review, we investigate the roles that stressors play in neurobehavioral development. We focus our review on the following forms of stress: psychosocial stressors (including anxiety, depression, material hardship, and emotional trauma), pathological stressors (including infectious and autoimmune diseases), and pharmacological stressors (namely, the use of opioids). Taking the fetal origins hypothesis in consideration, this review aims to explore how a wide range of maternal stressors can impact fetal neurodevelopment, as well as the mechanisms that govern these findings.

\footnotetext{
a Authors contributed equally to this work

b Authors contributed equally to this work.

c Authors contributed equally to this work.

d Authors contributed equally to this work.
} 
Given the overwhelming evidence for the link between maternal stressors and fetal neurodevelopment, this review also serves to emphasize the importance of prenatal screening for such stressors.

\section{Psychosocial Stressors}

Psychosocial stress is a prevalent issue among pregnant women. One study found that $23.6 \%$ of women experienced some level of anxiety, depression, and/or stress in their second trimester. ${ }^{2}$ As previously mentioned, it has long been hypothesized that maternal stress can negatively impact the fetus; thus, an investigation into the effects of psychosocial stressors on fetal development is warranted.

Davis et $\mathrm{al}^{3}$ investigated how maternal stress affects the baby's future ability (postpartum) to respond to (and recover from) stress. The researchers measured maternal cortisol levels during the late second and third trimesters of pregnancy as a quantification of maternal stress. Then, postpartum, each baby underwent a heel-stick blood draw 24 hours after birth, where the needle prick itself was meant to serve as a slightly painful stressor. After subjecting the baby to this stressor, researchers measured the baby's cortisol response as well as behavioral recovery time from the stress. Using growth curve analysis, mapping maternal and fetal cortisol levels as continuous variables, they found that higher maternal cortisol levels during pregnancy were associated with a higher cortisol response in the baby postpartum between 21 and 35 weeks of gestation $(P<.05) .{ }^{3}$ Davis et $\mathrm{al}^{3}$ also found a prolonged recovery time from the stressor: mothers with higher levels of perceived stress throughout pregnancy had infants with a significantly elevated level of arousal through the recovery period (from 13 to 35 weeks, $P<.05$ and between 36 and 38 weeks, $P<$ .10). ${ }^{3}$ In short, increased maternal stress during pregnancy was correlated with increased stress responses in the baby postpartum.

One potential confounding variable in the aforementioned study is that mothers who are more stressed in general may be more likely to partake in other activities that harm the fetus, including smoking. Tobacco exposure is a known risk factor for fetal developmental delay. ${ }^{4}$ However, a study by Rauh et $\mathrm{al}^{5}$ found that this risk was amplified if the mother also experienced "material hardship" (ie, psychosocial stress associated with not having adequate access to resources such as food, hygiene, and other materials). In this study, the authors administered a self-report survey of environmental tobacco smoke exposure and measure of material hardship. The mothers were also given air pollution monitors to wear. Infant growth data and a scale of infant intelligence was administered at 2 years of age to assess for neurodevelopmental delay. The authors found that the adverse effects of environmental exposure to tobacco was significantly greater in children born to mothers who reported greater material hardship $(P=.03)$, resulting in a deficit of around 7 points in the infant intelligence survey compared with infants born to mothers who were exposed to environmental tobacco and had less material hardship. ${ }^{5}$ In other 
words, the same "physical" stressor (tobacco and its byproducts) had worse effects on fetal neurodevelopment when the mother's psychosocial health was compromised. Thus, a link is established between maternal psychosocial health and fetal neurodevelopment. Such results should encourage physicians to not only screen for such physical stressors (ie, tobacco and alcohol use), but also to screen for socioeconomic status and material hardship, with the goal of mitigating these hardships to improve the health of their future child.

Emotional trauma, such as a sudden death in the family or an unexpected medical diagnosis, is another factor to consider when assessing the relationship between maternal psychosocial health and fetal neurodevelopment. Khashan et $\mathrm{al}^{6}$ investigated the offspring of women who experienced emotional trauma during (or slightly prior to) their pregnancy. In this study, mothers were said to have experienced emotional trauma if 1 or more close relatives died or received a diagnosis of cancer, stroke, or acute myocardial infarction up to 6 months before conception or during pregnancy. The cohort included more than 1 million mothers and their offspring and followed up the offspring from birth all the way through the first 10 years of life. The research suggested that the offspring of mothers who experienced emotional trauma during the first trimester of pregnancy were at higher risk (adjusted relative risk, 1.67 [95\% CI, 1.02-2.73]) for developing schizophrenia by their 10th year of life. ${ }^{6}$

Another source of maternal psychological stress is maltreatment in childhood. A study by Moog et $\mathrm{al}^{7}$ found that childhood maltreatment can have prolonged consequences that can be passed on from one generation to the next. Specifically, the researchers found that women who experienced childhood maltreatment were significantly more likely to have offspring with decreased intracranial volume $(P=.01$, adjusted $P=.02)$ and, therefore, increased risk for neurobehavioral abnormalities later in life. ${ }^{7}$ The study effectively controlled for confounders such as socioeconomic status, obesity, and complications during delivery, thus supporting the notion that maternal emotional trauma is associated with impaired neurodevelopment in utero. ${ }^{7}$

At this point, it appears that the maternal psychological state has the potential to impact fetal neurodevelopment. However, the mechanisms by which these phenomena occur are not as well understood. Multiple studies of the physiology of maternal stress and its impact on fetal neurodevelopment have focused on the hypothalamic-pituitary-adrenal axis, which is a key player in the induction and regulation of stress. ${ }^{3,8,9}$ During the stress response, the hypothalamus releases corticotropin-releasing hormone, which signals the release of adrenocorticotropic hormone from the anterior pituitary gland, which in turn signals the release of glucocorticoids (especially cortisol) from the adrenal gland. ${ }^{10} \mathrm{~A}$ study by Evans et $\mathrm{al}^{11}$ found that pregnant women with both anxiety and depression had significantly higher cortisol levels compared with those in the control group $(P=.006)$. 
A logical question follows: How much of the maternal cortisol can actually reach the fetal circulation? While the fetus is known to produce an enzyme called 11- $\beta$-hydroxysteroid dehydrogenase 2 that blocks the entry of maternal cortisol into fetal circulation, some cortisol is still able to pass through to the fetus. ${ }^{12}$ Glover et al ${ }^{12}$ showed that mothers in the highest quartile of the Spielberger State-Trait Anxiety Inventory had significantly elevated cortisol levels compared with those in the lowest quartile $(P<.002)$. This increase in cortisol has the potential to have lasting impacts on fetal neurodevelopment. ${ }^{3}$ Research to understand whether there is dose-dependent harm done based on increased cortisol levels could have important clinical implications.

In addition to the hypothalamic-pituitary-adrenal axis/cortisol hypothesis, other mechanisms have been proposed to explain the effects of maternal psychosocial distress on fetal neurodevelopment. Rakers et $\mathrm{al}^{13}$ reported that cortisol by itself cannot explain the breadth of effects that maternal stress has on fetal development, and that multiple pathways are actually at play. First, the study proposed the idea that high maternal stress would lead to high levels of catecholamines (namely, epinephrine and norepinephrine), which would induce vasoconstriction of the uterine artery and thus decreased perfusion to the uterus. Second, the study proposed that maternal stress may alter the maternal gut microbiome, which is known to play a role in early brain development (a phenomenon known as the gut-brain axis). ${ }^{13}$ More studies on these alternate hypotheses are needed to paint a more comprehensive picture of the relationship between maternal psychosocial health and early neurodevelopment.

\section{Pathological Stressors}

\section{Infection}

Multiple research studies have elucidated the detrimental effects of in utero infection on postpartum neurological function and anatomical development. A retrospective case-control study found that some mental disorders may, in part, be due to fetal exposure to infection during the second trimester in pregnancy. ${ }^{14}$ Machón et $\mathrm{a}^{14}$ found a significant increase in the hospital diagnosis rates for major affective disorder, specifically the unipolar forms, for individuals exposed to the 1957 influenza epidemic during their second trimester of fetal development compared with controls.

In addition to major affective disorder, prenatal influenza infection, caused by a viral source, has been linked to the development of adult schizophrenia. In a retrospective case-control study, Brown et $\mathrm{al}^{15}$ found a 7 -fold increased risk of developing schizophrenia in individuals who were exposed to viral influenza infection in their first trimester of development Interestingly, when combined with the results of the Machón et a ${ }^{14}$ study, these data suggest that exposure to influenza at different points in fetal development can lead to different neurodevelopmental disorders. It is unclear whether this difference in timed 
exposure and neurodevelopmental consequences is related to the specific strain of influenza, underlying neurodevelopmental pathophysiology, or some other confounding effect.

Mortensen et $\mathrm{al}^{16}$ found a moderate yet non-significant association with fungal Toxoplasma gondii infection in utero and schizophrenia, suggesting that the effect is not strain specific. When looking at 63 individuals who developed schizophrenia spectrum disorders, the group's studies revealed an increased risk (odds ratio, 2.61 [95\% CI, 1.00-6.82]) of mothers having IgG antibodies to T gondii. ${ }^{16}$ Many possible confounders exist, yet one possible explanation for this result could be that the development of schizophrenia is, in part, due to an inflammation-mediated process, although a 2017 genome-wide association study suggested a 2 -fold elevation in C-reactive protein level may actually be protective against schizophrenia $(0.90$ [95\% CI, 0.84-0.97]; $P=.005) .{ }^{17}$ Further research is needed to clarify this hypothesis.

Not only is in utero exposure to infection related to the development of schizophrenia, but one study found a possible link between these infections and deficits in executive function. ${ }^{18}$ The study looked at 26 people with diagnosed schizophrenia and their "...performance on the Wisconsin Card Sorting Test and the Trail Making Test, part B (Trails B), as well as other measures of executive function[.]"18 The study results showed that patients with schizophrenia who were "...exposed in utero to infection committed significantly more total errors on the WCST and took significantly more time to complete the Trails B than unexposed cases." ${ }^{18}$ Future research should aim to identify the extent of cognitive function deficits related to exposure to infection during gestation.

The neurodevelopmental structural consequences of prenatal infection have been studied in the context of schizophrenia. Brain magnetic resonance images were acquired of patients diagnosed as having schizophrenia, and the length of the cavum septum pellucidum (CSP) was compared between patients with and without serologically confirmed exposure to influenza in utero. ${ }^{19} \mathrm{~A}$ linear regression was performed with CSP length as the dependent variable and the indicator for exposed-unexposed status as a predictor variable. Using a 2-tailed test, a statistically significant increase in mean length of the CSP was found in schizophrenia cases exposed to in utero infection compared with those who were unexposed. ${ }^{19}$ These data support the hypothesis that infection in utero has direct neurodevelopmental and anatomical consequences that persist postnatally.

\section{Maternal Autoantibody Flares}

Previous experimental and observational studies have shown a strong correlation between maternal rheumatic diseases (as well as other autoimmune diseases) and the development of psychiatric disorders among offspring, such 
as obsessive-compulsive disorder ${ }^{20}$ Tourette syndrome, ${ }^{21}$ and autism spectrum disorders (ASD). ${ }^{22}$ These findings support the fetal origins hypothesis.

The nature of fetal immunity and acquired immunity after birth mandates that maternal IgG antibodies cross the placenta and enter into fetal circulation. ${ }^{23}$ Current literature suggests that among the maternal antibodies entering the placental circulation and crossing the fetal blood-brain barrier are those that are present during flares in autoimmune diseases. ${ }^{24}$ These autoantibodies have been marked as the culprit for the neurotoxic basis of learning disabilities that present in children born to mothers with autoimmune conditions. ${ }^{25}$

ASDs have rapidly become the most common neurodevelopmental disorder, with 1 in 88 individuals receiving a diagnosis. ${ }^{26}$ The spectral diseases have an unknown etiology, although the role of maternal stress elucidates a potential molecular etiology of these and other related neurological disorders. ${ }^{26}$

The research into maternal autoantibodies and their involvement in neuropsychological disorders, such as ASDs, was prompted by a strong epidemiologic basis. ${ }^{27}$ One study ${ }^{27}$ demonstrated that in a given cohort of mothers with systemic lupus erythematosus, 1 in 4 children born was found to have a learning disability. The children were assessed based on a modified version of the Edinburgh Hand Preference Questionnaire, as well as standardized tests of reading, arithmetic, and writing achievement. These tests showed that the children born to mothers with systemic lupus erythematosus scored 1.5 SDs below the mean. This study was conclusive and emphasized the importance of future research into decreasing the levels of maternal autoantibodies to reduce the disease burden in the mother and prevent cognitive impairment in the future child. ${ }^{27}$

Observable abnormalities in murine fetal brain development were seen in a study conducted to find evidence of the molecular maternal involvement of lupus antibodies in cortical development in mice. ${ }^{25}$ The study observed that mothers measured with high titers of these autoantibodies correlated with offspring with cognitive impairment, which was quantified by histological abnormalities in the fetal brain and diminished performance on tests. The autoantibodies in lupus-affected mothers specifically target the N-methyl-Daspartate receptor; thus, the study went on to inject high levels of $\mathrm{N}$-methylD-aspartate receptor autoantibodies into gravid mice to measure a direct neurotoxic effect in the fetal brains. When the mice offspring were born, they were put through a series of controlled cognitive challenges, in which the experimental mouse group performed significantly worse. ${ }^{25}$

Currently, there are several potential mechanisms suggested to explain the autoantibodies' effects on fetal neurodevelopment. The first claims that the antibody functions as an agonist of a receptor or signaling cascade that initiates inflammation and hinders neurological development. ${ }^{26}$ The antibodies alone 
could also be initiating the inflammation, which negatively impacts the developmental progress. It has also been suggested that developmental cascades are being blocked and the target molecules are unable to function as intended. $^{26}$

What is observable, however, is a trend of high titers of antibodies inducing progressively worse impairment in fetal neurodevelopment. Thus, maternal autoimmune disease flares must be carefully monitored, and more research into the exact mechanism is required to elucidate the process of antibody effects on fetal neurodevelopment.

\section{Pharmacological Stressors}

When considering the etiology of maternal and fetal stress, many consider traditional factors such as psychosocial factors, socioeconomic factors, chronic disease, and nutritional status. However, the significant amount of pharmacological development that has occurred in the past 30 years has added a further consideration for maternal and fetal stress: drugs and medicines.

While the discovery of synthetic opioids, such as oxycodone, occurred in the early 20th century, widespread marketing and prescription of synthetic opioids were not prevalent in the United States until the Food and Drug Administration approved OxyContin for analgesic use in $1995 .{ }^{28}$ Under Food and Drug Administration guidelines, OxyContin and other opioid analgesics were approved for use among patients with moderate to severe chronic pain, including pregnant women. ${ }^{28}$ In 2015 , Desai et $\mathrm{al}^{29}$ investigated trends in opioid use during pregnancy in US women. The investigators found that in the 1.1 million women enrolled in the study, $21.6 \%$ had been prescribed and filled a prescription for an opioid analgesic during pregnancy. ${ }^{29}$ Despite high rates of opioid analgesic use in pregnant women, the consequences of prepartum opioid exposure for the developing fetus are currently not well elucidated. In response to such concerns, several studies have investigated the effects of fetal opioid exposure on neurodevelopment and future behavioral development.

During early human fetal development, the neural tube serves as the embryonic precursor to the central nervous system (CNS), consisting of the brain and spinal cord. ${ }^{30}$ Much of neural tube development occurs in the first month following fertilization, including time periods in which a woman may not be aware of the current pregnancy. ${ }^{30}$ In 2013, Yazdy et al ${ }^{31}$ investigated potential associations between maternal opioid use during the periconceptional period and neural tube defects in offspring. Study participants were identified from the ongoing Slone Epidemiology Center Birth Defects Study. Two study groups were identified for the offspring: experimental group candidates included infants with encephalocele, anencephaly, and/or spina bifida and control group candidates had no major malformations. The mothers of these infants were interviewed for demographic information, relevant medical history, social behaviors, and medication use. Subsequently, both groups were 
analyzed for distribution of opioid exposures and stratified based on sociodemographic factors. The investigators found that the offspring of mothers with reported opioid use had a significantly higher rate of neural tube defects. ${ }^{31}$ Extrapolation of the data collected in this study revealed that periconceptional opioid exposure lead to a 2.2-fold increase in neural tube defects, with overall prevalence of 5.9 neural tube defects per 10000 live births in mothers taking opioids. ${ }^{31}$ Overall, the findings of this study support an association between periconceptional maternal opioid use and increased risk of neural tube defects; however, the investigators of the study acknowledged the inability to form a dose-dependent relationship between opioid analgesics and rates of neural tube defects. ${ }^{31}$ The database used for data collection did not include information regarding the dosage of the opioid analgesics that were prescribed, nor did it include the duration of opioid analgesic use. ${ }^{31}$ Despite the limitations, these findings are concerning and indicate the need for further investigation of the safety of opioid analgesic use during the periconceptional period.

One of the central dogmas of embryology is that structure and function of a tissue or organ are often interrelated such that a defect in the structure of a tissue often leads to functional deficits. With the understanding that opioid analgesics have the potential to cause structural defects of the neural tube and developing CNS, it is supposed that periconceptional opioid use will lead to an increased risk for functional deficits of the CNS, such as cognitive developmental delay.

While a number of studies have examined the structural and anatomical consequences for fetuses of mothers with periconceptional opioid use, it is also crucial to examine the effects of periconceptional opioid use on the neurodevelopment of such offspring. In the literature, the steady-state visual evoked potential has been used as an assay for measuring neurodevelopment in human infants. ${ }^{32}$ This assay investigates visual evoked potentials, which are physiological responses to visual field stimulation at specific frequencies of light. In response to excitation from this visual stimulus, the CNS develops electrical activity in multiples of the frequency to which the retina was previously exposed. ${ }^{32}$ The ability to develop these visually evoked potentials is a hallmark of neurodevelopment. ${ }^{32}$ In 2008 , McGlone et al ${ }^{32}$ investigated the characteristics of visual evoked potentials in infants born to mothers taking a course of methadone for treatment of illicit opioid use. Infant participants in the study were recruited from a subset of patients in the postnatal wards of a number of hospitals in Glasgow, Scotland. Two groups were established for this study: a control group of infants without methadone exposure and an experimental group of infants with periconceptional methadone exposure.

For this study, visual evoked potentials were measured at 4 days and 1 week following birth. In addition to neurodevelopmental data, investigators collected demographic information, birth weight, and occipitofrontal 
circumference. The investigators found that the infants born to mothers receiving methadone treatment had significantly lower birth weight and occipitofrontal circumference compared with the control infants. ${ }^{32}$ In addition, the methadone-exposed infants were found to have significantly higher rates of visual evoked potentials with abnormal waveforms as well as higher rates of low-amplitude visual evoked potentials. ${ }^{32}$ Such findings are consistent with the theory that periconceptional opioid exposure is associated with defects in visual development in infants. Overall, the literature demonstrates that periconceptional opioid use is associated with an increased risk for neurodevelopmental delay. While it has been shown that opioids cross the placenta ${ }^{11}$ and can be detected in the umbilical cord, placenta, and meconium, the effects of exposure to opioids on the developing human fetus are unknown. ${ }^{12,30}$

\section{Conclusions}

The literature supports that a wide range of maternal stressors have the potential to negatively impact the neurobehavioral development of the fetus, the future baby, and the adult. Psychosocial stressors, including anxiety, depression, material hardship, and emotional trauma, have been associated with adverse neurodevelopmental outcomes. Likewise, pathological stressors, such as infection and autoimmune disease, have been shown to have neurotoxic effects on the developing fetus and future baby.

In addition, the use of opioids among pregnant women has been associated with both anatomical and neurodevelopmental abnormalities. These studies provide strong support for the fetal origins hypothesis because we see that the uterine environment can have profound effects on the fetus and future child. The evidence and correlations in these studies merit a greater focus on prenatal screening for maternal stressors, including those related to psychosocial stress, disease state, and medication use. These screening measures could lead to a better understanding of the mechanisms that affect fetal growth. There may be potential for preventive efforts with further screening as well as protocol development to better manage high-risk mothers and fetuses. Further, we found it difficult to ignore the dearth of information concerning maternal stress during pregnancy, and we found a need to better quantify different stressors and their nuanced degrees of severity. This would improve the understanding of mechanisms that will elucidate connection between maternal stress and the various neurobiologies of uterine environmental factors.

Stress on the maternal body can directly impact the fetal brain and lead to lasting changes in its development and function. In an era where mental health is an increasingly pressing issue, this is especially important to consider. We emphasize that prenatal screening efforts should include a wide range of maternal stressors, and that pregnant patients should be advised on stress management to control for adverse neurological effects in the fetus. 


\section{REFERENCES}

1. Almond D, Currie J. Killing me softly.J Econ Perspect. 2011;25:153-172.

2. Nagandla K, Nalliah S, Yin L, et al. Prevalence and associated risk factors of depression, anxiety and stress in pregnancy. Int J Reprod Contracept Obstet Gynecol. 2016;5:2380-2388.

3. Davis EP, Glynn LM, Waffarn F, Sandman CA. Prenatal maternal stress programs infant stress regulation. JChild Psychol Psych. 2011;52:119-129.

4. Kahn RS, Khoury J, Nichols WC, Lanphear BP. Role of dopamine transporter genotype and maternal prenatal smoking in childhood hyperactive-impulsive, inattentive, and oppositional behaviors. J Pediatr. 2003;143:104-110.

5. Rauh VA, Whyatt RM, Garfinkel R, et al. Developmental effects of exposure to environmental tobacco smoke and material hardship among inner-city children. Neurotoxicol Teratol.

2004;26:373-385.

6. Khashan AS, Abel KM, McNamee R, et al. Higher risk of offspring schizophrenia following antenatal maternal exposure to severe adverse life events. Arch Gen Psychiatry. 2008;65:146-152.

7. Moog NK, Entringer S, Rasmussen JM, et al. Intergenerational effect of maternal exposure to childhood maltreatment on newborn brain anatomy. Biol Psychiatry. 2018;83:120-127.

8. Gutteling BM, De Weerth C, Buitelaar JK. Maternal prenatal stress and 4-6 year old children's salivary cortisol concentrations pre- and post-vaccination. Stress. 2004;7(4):257-260.

9. Gutteling BM, Weerth C, Buitelaar JK. Prenatal stress and children's cortisol reaction to the first day of school. Psychoneuroendcrinology. 2005;30(6):541-549.

10. Smith SM, Vale WW. The role of the hypothalamic-pituitary-adrenal axis in neuroendocrine responses to stress. Dialogues Clin Neurosci. 2006;8:383-395.

11. Evans LM, Myers MM, Monk C. Pregnant women's cortisol is elevated with anxiety and depression - but only when comorbid. Arch Womens Ment Health. 2008;11:239.

12. Glover V, Bergman K, Sarkar P, O’Connor TG. Association between maternal and amniotic fluid cortisol is moderated by maternal anxiety. Psychoneuroendocrinology. 2008;34:430-435.

13. Rakers F, Rupprecht S, Dreiling M, Bergmeier C, Witte OW, Schwab M. Transfer of maternal psychosocial stress to the fetus. Neuroscience E Biobehavioral Reviews. February 2017. doi:10.1016/ j.neubiorev.2017.02.019

14. Machón RA, Mednick SA, Huttunen MO. Adult major affective disorder after prenatal exposure to an influenza epidemic. Arch Gen Psychiatry. 1997;54:322-328.

15. Brown AS, Begg MD, Gravenstein S, et al. Serologic evidence of prenatal influenza in the etiology of schizophrenia. Arch Gen Psychiatry. 2004;61:774-780.

16. Mortensen PB, Nørgaard-Pedersen B, Waltoft BL, Sørensen TL, Hougaard D, Yolken RH. Early infections of Toxoplasma gondii and the later development of schizophrenia. Schizophrenia Bulletin. 2007;33:741-744.

17. Hartwig FP, Borges MC, Horta BL, Bowden J, Davey Smith G. Inflammatory biomarkers and risk of schizophrenia: A 2-sample mendelian randomization study. JAMA Psychiatry.

2017;74(12):1226-1233. 
18. Brown AS. Exposure to prenatal infection and risk of schizophrenia. Front Psychiatry. 2011;2:63.

19. Brown AS, Deicken RF, Vinogradov S, et al. Prenatal infection and cavum septum pellucidum in adult schizophrenia. Schizophr Res. 2008;108:285-287.

20. Dale RC, Heyman I, Giovannoni G, Church AW. Incidence of anti-brain antibodies in children with obsessive-compulsive disorder. Br J Psychiatry. 2005;187:314-319.

21. Dale RC. Post-streptococcal autoimmune disorders of the central nervous system. Dev Med Child Neurol. 2005;47:785-791.

22. Enstrom AM, Van de Water JA, Ashwood P. Autoimmunity in autism. Curr Opin Investig Drugs. 2009;10:463-473.

23. Vinet É, Bernatsky S. Outcomes in children born to women with rheumatic diseases. Rheum Dis Clin North Am. 2016;43:263-273.

24. Brimberg L, Sadiq A, Gregersen PK, Diamond B. Brain-reactive IgG correlates with autoimmunity in mothers of a child with an autism spectrum disorder. Mol Psychiatry. 2013;18:1171-1177.

25. Lee JY, Huerta PT, Zhang J, et al. Maternal lupus and congenital cortical impairment. Nature Medicine. 2008;15:91-96.

26. Braunschweig D, Water J. Maternal autoantibodies in autism. Arch Neurol. 2012;69:693-699.

27. Ross G, Sammaritano L, Nass R, Lockshin M. Effects of mothers' autoimmune disease during pregnancy on learning disabilities and hand preference in their children. Arch Pediatr Adolesc Med. 2003;157:397-402.

28. OxyContin. Xtampza ER (oxycodone) dosing, indications, interactions, adverse effects, and more. 2018. https://reference.medscape.com/drug/oxycontin-xtampza-er-oxycodone-343321. Accessed April 30, 2019.

29. Desai RJ, Hernandez-Diaz S, Bateman BT, Huybrechts KF. Increase in prescription opioid use during pregnancy among Medicaid-enrolled women. Obstetric Anesthesia Digest. 2015;35(2):59-60. doi:10.1097/01.aoa.0000463803.54491.04

30. Gilbert SF. Formation of the Neural Tube. In: Developmental Biology. 6th ed. Sinauer Associates; 2000. https://www.ncbi.nlm.nih.gov/books/NBK10080/. Accessed April 30, 2019.

31. Yazdy MM, Mitchell AA, Tinker SC, Parker SE, Werler MM. Periconceptional use of opioids and the risk of neural tube defects. Obstetrics E Gynecology. 2013;122(4):838-844. doi:10.1097/ aog. $0 \mathrm{~b} 013 \mathrm{e} 3182 \mathrm{a} 6643 \mathrm{c}$

32. McGlone L, Mactier H, MacKinnon JR. Outcome in infants exposed to methadone in utero. BMJ. 2008;337(sep23 2):a1774-a1774. doi:10.1136/bmj.a1774 